\title{
Management of acute meningitis
}

\author{
Authors: Michael J Griffiths, ${ }^{A, B, C}$ Fiona McGill ${ }^{D, E, F}$ and Tom Solomon ${ }^{G, H, I}$
}

\section{Acute meningitis remains a devastating disease. Clinicians need a low threshold for suspecting meningitis, to undertake appropriate investigations and provide treatment in a timely manner, to minimise the risk of poor outcome in bacterial} disease, while limiting unnecessary treatment in viral meningitis.

\section{Definitions}

Meningitis is inflammation of the meninges covering the brain. It is a pathological definition. The cerebrospinal fluid (CSF) typically exhibits an elevated number of leucocytes (or a pleocytosis). In adults, $>5$ leucocytes $/ \mu \mathrm{L}$ is defined as elevated. Bacterial or viral meningitis is confirmed by the detection of a pathogen in the CSF. Bacterial meningitis may also be suggested by symptoms of meningism and appropriate bacteria in the blood. ${ }^{1}$

\section{Causes}

The most common causes of meningitis in immunocompetent adults in the UK are viruses and bacteria. Viruses account for up to half of cases. Enterovirus is the commonest, with herpes simplex and varicella zoster the next most frequent. Streptococcus pneumoniae and Neisseria meningitidis are the commonest bacteria, together accounting for approximately one-quarter of cases.

Other causes such as Haemophilus influenzae, Listeria monocytogenes, Mycobacterium tuberculosis and fungi (typically cryptococci) are less frequently detected, together representing

Authors: ${ }^{\text {A }}$ senior clinical lecturer, Institute of Infection and Global Health, University of Liverpool, Liverpool, UK; ${ }^{\text {B }}$ honorary consultant in paediatric neurology, Alder Hey Children's NHS Foundation Trust, Liverpool, UK; ' National Institute of Health Research Health Research Protection Unit in Emerging and Zoonotic infections, UK; ${ }^{D}$ clinical lecturer, Institute of Infection and Global Health,

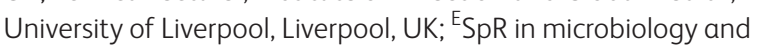
infectious diseases, Royal Liverpool and Broadgreen University Hospitals NHS Trust, Liverpool, UK; ' F National Institute of Health Research Health Research Protection Unit in Emerging and Zoonotic infections, UK; ${ }^{G}$ professor of neurological sciences, Institute of Infection and Global Health, University of Liverpool, Liverpool, UK; ${ }^{\text {H}}$ honorary consultant in neurology, The Walton Centre NHS Foundation Trust, Liverpool, UK; ${ }^{\mathrm{I}}$ National Institute of Health Research Health Research Protection Unit in Emerging and Zoonotic infections, UK
$<10 \%$ of cases. Currently, many adults with meningitis have no pathogen detected. ${ }^{2-5}$

Although this article refers to Listeria and tuberculous meningitis, it purposely focuses on the more common causes, ie viral and bacterial meningitis.

\section{Clinical features}

Clinical features alone cannot confirm the diagnosis of meningitis. A lumber puncture (LP) is essential to confirm the diagnosis of meningitis and establish the cause.

In one study, $95 \%$ of bacterial meningitis patients had at least two symptoms of headache, neck stiffness, fever and altered consciousness. The latter three features were present together in only $44 \%$ of cases. Neurological deficits are found in around onethird of patients. ${ }^{6}$ Similar findings are reported by other studies. ${ }^{7-9}$

A rash in suspected meningitis makes $N$ meningitidis more likely. However, $37 \%$ of meningococcal meningitis patients have no rash. $^{8}$ Varicella and enterovirus can also be associated with a rash.

Risk factors for Listeria meningitis include overt or relative immune compromise, the latter including chronic illness, diabetes, alcohol dependency, malignancy or old age. Listeria

\section{Key points}

Viral meningitis is the most common form of meningitis in the UK, but bacterial meningitis continues to be important, with a high mortality

Clinical features are poor discriminators for meningitis, so urgent investigations, starting with lumbar puncture, are key

Most patients do not need brain imaging before lumbar puncture. Patients exhibiting clinical features of brain shift warrant urgent CT. Otherwise, imaging can cause delays in commencing antibiotics, which can lead to increased mortality

Aim to take $10 \mathrm{~mL}$ of CSF during LP. Larger volumes are especially useful to diagnose tuberculous meningitis, and enable additional aliquots to be available for further diagnostic testing

Prompt testing of CSF and blood by PCR can hasten pathogen diagnosis and improve patient management

KEYWORDS: clinical, viral, bacterial, meningitis, management, diagnostics 
meningitis is rarely seen in immunocompetent adults under 60 years of age. ${ }^{1,10}$

Travel history, symptoms of otitis media / sinusitis, contact with another person with meningitis, sepsis or tuberculosis are other useful diagnostic clues.

\section{Investigations}

\section{Lumbar puncture}

Lumbar puncture is the key investigation. It enables rapid confirmation of meningitis and type of infecting organism. Diagnostic yield of LP can be diminished by collecting small CSF volumes. At least $10 \mathrm{~mL}$ can be safely removed. ${ }^{11,12}$

\section{Cerebrospinal fluid cell count}

Cerebrospinal fluid remains one of the most rapidly informative tests. Pleocytosis indicates meningeal inflammation, of which infection is the most common cause. Van de Beek and colleagues reported that $>90 \%$ of adults with bacterial meningitis had a CSF leukocyte count $>100$ cells/ $\mathrm{LL} .{ }^{6}$ Absence of pleocytosis makes meningitis much less likely, but does not completely rule it out. Approximately $1-2 \%$ of patients with bacterial meningitis will have a normal CSF leukocyte count. Positive pathogen detection and an absence of pleocytosis more frequently occurs among children, the immunocompromised, those pretreated with antibiotics or with mycobacteria tuberculosis infection. ${ }^{13}$

\section{Cerebrospinal fluid leukocyte differential}

Cerebrospinal fluid leukocyte differential can help predict which type of pathogen is causing infection. Lymphocyte predominance suggests viral, while neutrophil predominance suggests bacterial infection. There are several exceptions to this general guide, including CSF neutrophil predominance observed in association with tuberculous meningitis (Table 1).

\section{Cerebrospinal fluid biochemistry}

Cerebrospinal fluid glucose is normally approximately two-thirds the blood (plasma) concentration. It is often lower in bacterial and tuberculous meningitis. As CSF glucose is influenced by the plasma glucose, it is essential to measure blood glucose at LP, to obtain an accurate CSF:blood glucose ratio. A CSF:blood glucose ratio $<0.36$ is an accurate $(93 \%)$ marker for distinguishing bacterial from viral meningitis. ${ }^{1}$

Cerebrospinal fluid protein is normally $<0.4 \mathrm{~g} / \mathrm{L}$. Elevated protein suggests inflammation. A CSF protein $<0.6 \mathrm{~g} / \mathrm{L}$ largely rules out bacterial infection. ${ }^{1}$

\section{Cerebrospinal fluid parameters}

Cerebrospinal fluid parameters have been combined into tools to help diagnose bacterial meningitis. One prediction rule accurately distinguished bacterial from viral meningitis in two adult patient populations using retrospective data (area under the curve 0.97). ${ }^{14,15}$ Clinical prediction tools (using CSF, laboratory and clinical parameters) have also exhibited high accuracy when tested retrospectively in large child populations. ${ }^{16}$ No tools have been validated prospectively in adults in the UK.

\section{Pathogen detection}

\section{Cerebrospinal fluid microscopy with Gram stain}

Cerebrospinal fluid microscopy with Gram stain (or an acid fast stain for $M$ tuberculosis) can rapidly detect bacteria. It has a sensitivity between $50 \%$ and $99 \% .{ }^{6}$ Detection, particularly for $M$ tuberculosis, is enhanced by collection of $>10 \mathrm{~mL}$ of CSF and subsequent cytospin. ${ }^{11}$

\section{Cerebrospinal fluid culture}

Cerebrospinal fluid culture is historically regarded as the 'gold standard' for the diagnosis of bacterial meningitis. It is diagnostic in $70-85 \%$ of cases prior to antibiotic exposure. Sensitivity decreases by $20 \%$ following antibiotic pretreatment. Cerebrospinal fluid sterilization can occur within 2-4 hours of antibiotic administration for meningococci and pneumococci respectively. ${ }^{15}$ Lumbar puncture should be performed as soon as possible to maximise pathogen detection.

Table 1. Classical cerebrospinal fluid features for different causes of meningitis ${ }^{a}$

\begin{tabular}{|c|c|c|c|c|c|}
\hline & Normal & Bacterial & Viral & Tuberculous & Fungal \\
\hline Opening presssure (cm CSF) & $12-20$ & Raised & Normal / mildly raised & Raised & Raised \\
\hline Appearance & Clear & Purulent, turbid, cloudy & Clear & Clear or cloudy & Clear or cloudy \\
\hline 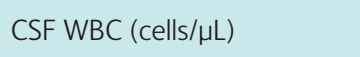 & $<5$ & Raised $(>100)^{b}$ & Raised $(5-1000)^{b}$ & Raised $(5-100)^{b}$ & Raised $(5-100)^{b}$ \\
\hline Predominant cell & $\mathrm{n} / \mathrm{a}$ & Neutrophils ${ }^{c}$ & Lymphocytes $^{d}$ & Lymphocytes $^{e}$ & Lymphocytes \\
\hline CSF protein $(\mathrm{g} / \mathrm{L})$ & & Raised & Mildly raised & Markedly raised & Raised \\
\hline CSF plasma glucose ratio & $>0.66$ & Very low & Normal / slightly low & Very low & Low \\
\hline CSF glucose (mmol) & $2.6-4.5$ & Very low & Normal / slightly low & Very low & Low \\
\hline \multicolumn{6}{|c|}{$\begin{array}{l}\text { Note: Local laboratory ranges for biochemical tests should be consulted. They may vary from the quoted values here. A traumatic lumbar puncture (LP) will affect the } \\
\text { results by falsely elevating the white cells due to excessive red cells. A common correction factor used is 1:1000. } \\
\text { aDerived from Tom Solomon-Lecture Notes in Neurology } \\
\text { b Occasionally the CSF white cell count (WCC) may be very high (several thousand) in bacterial meningitis. Alternatively the CSF WCC may be normal (especially in } \\
\text { immunodeficiency or tuberculous meningitis). } \\
\text { 'May be lymphocytic if antibiotics given before LP (partially treated bacterial meningitis), or with certain bacteria, eg Listeria monocytogenes. } \\
\text { dMay be neutrophilic in enteroviral meningitis (especially early in disease). } \\
\text { eMay be neutrophilic early in the disease course. } \\
\text { CSF = cerebrospinal fluid; WBC = white blood cell count; WCC = white cell count }\end{array}$} \\
\hline
\end{tabular}




\section{Cerebrospinal fluid polymerase chain reaction}

Cerebrospinal fluid polymerase chain reaction (PCR), using pathogen specific nucleic acid sequences, can detect both bacteria and viruses with high sensitivity. Polymerase chain reaction is the 'gold standard' for diagnosis of viral meningitis. Polymerase chain reaction is increasingly relied upon in bacterial meningitis. It has far greater sensitivity than culture in invasive meningococcal disease. ${ }^{17}$ Cerebrospinal fluid PCR is particularly valuable in patients who receive antibiotics before LP. Polymerase chain reaction for $16 \mathrm{~S}$ ribosomal RNA (present in almost all bacteria) enables a broad screen for bacteria, but has lower sensitivity than pathogen specific PCR. ${ }^{1,15}$

\section{Blood tests}

Blood cultures should always be taken on admission and are helpful when antibiotics are started before LP. Blood cultures are positive in $50-80 \%$ of bacterial meningitis cases. ${ }^{15}$

Blood PCR is increasingly important, especially as PCR detects bacteria several days after antibiotic initiation. ${ }^{17}$ Blood PCR substantially increases the confirmation in meningococcal disease. ${ }^{18}$

Despite these tests, many patients will not have a cause identified for their meningitis.

Blood biomarkers, such as procalcitonin and C-reactive protein, can help distinguish bacterial from viral meningitis in adults and can be used to help guide treatment if no aetiology is found. ${ }^{19,20}$ Host biomarkers for detecting bacterial meningitis are being actively investigated by our Liverpool group and others. To date, there is insufficient evidence to recommend their routine use in the NHS. ${ }^{1}$

\section{Swabs}

Throat, nasopharyngeal, and stool swabs are useful for detecting enteroviruses if the CSF PCR is negative.

\section{Brain imaging}

Brain imaging is neither obligatory in the management of meningitis, nor a prerequisite to LP. Performing neuroimaging before LP is associated with delays in commencing antibiotics, which in turn can lead to an increase in mortality. ${ }^{12,21}$ An urgent CT scan should be performed if there are clinical signs of brain shift. Clinical features indicative of a brain shift include focal neurological signs and reduced Glasgow Coma Score (GCS) (Box 1). The 2016 UK meningitis guidelines recommend an LP be performed without prior neuroimaging if the GCS is $>12$. Patients with a GCS $\leq 12$ should be considered for critical care,

\begin{tabular}{l} 
Box 1. Indications for brain imaging before lumbar \\
puncture (LP) in suspected meningitis ${ }^{a}$ \\
$>$ Focal neurological signs \\
$>$ Presence of papilloedema ${ }^{b}$ \\
$>$ Continuous or uncontrolled seizures GCS12 \\
\hline${ }^{a}$ To exclude significant brain swelling or shift that may predispose to cerebral \\
herniation post LP. \\
${ }^{b}$ Inability to view the fundus is not a contraindication to LP, especially in \\
patients who have had a short duration of symptoms. \\
CLumbar puncture without prior neuroimaging may be safe at levels below this. \\
GCS = Glasgow Coma Score
\end{tabular}

intubation assessment and neuroimaging. Imaging, particularly when contrast is used, may exhibit meningeal enhancement in meningitis. When brain shift is identified liaison with critical care and neurosurgical teams are essential.

A summary of investigations is presented in Fig 1.

\section{Treatment}

If a patient exhibits signs of airway, breathing or circulatory difficulties (eg in coexisting sepsis), management should initially focus on stabilisation of these systems.

All patients should be reviewed by a senior clinician. The Royal College of Physicians recommends consultant review for all acute medical patients within 14 hours of admission. Urgency of review should be assessed using the National Early Warning Score. The GCS should be recorded for its prognostic value, and to enable changes to be monitored. Presence of a rash and use of preadmission antibiotics should also be recorded.

If the patient presents with sepsis, they should be managed according to the sepsis guidelines. ${ }^{22}$ If the infective focus of sepsis is meningitis, then the antibiotic treatment should follow the guidelines for meningitis. ' For example, piperacillin/ tazobactam is not recommended for use in sepsis secondary to meningitis, because of its poor penetration of the blood brain barrier. A recent large open-label trial showed no benefit of prehospital antibiotics in sepsis. ${ }^{23}$ Previous trials for meningitis have also been inconclusive. ${ }^{1}$ Consequently, the benefit of prehospital antibiotics for suspected meningitis is unclear. Management of other aspects of sepsis, eg circulation, should follow the sepsis guidelines. ${ }^{22}$

Treatment for bacterial meningitis is antibiotics, with or without steroids. The choice of antibiotics is a three stage process: an initial empirical decision based on clinical suspicion, review following microscopy results, and review again when culture or PCR results are available (Fig 2).

In suspected bacterial meningitis, dexamethasone should be started either shortly before or simultaneously with antibiotics at $10 \mathrm{mg}$ intravenously (IV) 6-hourly. Up until 12 hours after antibiotic initiation, dexamethasone can still be started, but the impact of this on mortality has not been studied. If pneumococcal meningitis is probable, dexamethasone should continue for 4 days. In suspected tuberculous meningitis, dexamethasone provision should follow the recommended guidelines. ${ }^{11}$ Once another cause of meningitis is probable, dexamethasone should be stopped.

There is no specific treatment for viral meningitis. Treatment with aciclovir has only been of proven benefit in herpes encephalitis, not meningitis. Only if the patient has encephalitic features, such as impairment of consciousness, focal neurological signs, inflammation of brain parenchyma in the region of the temporal lobe on cranial imaging, should aciclovir be considered.

\section{Prognosis}

Mortality occurs almost exclusively in bacterial meningitis. Up to $57 \%$ in meningococcal sepsis, $30 \%$ of pneumococcal and $7 \%$ of meningococcal meningitis without sepsis cases die.,

Morbidity is common in bacterial meningitis. Van De Beek's study reported focal neurological deficits in $50 \%$ of cases, the commonest being hearing impairment (14\%), and $14 \%$ of cases exhibited moderate to severe disability at discharge. ${ }^{6}$ 


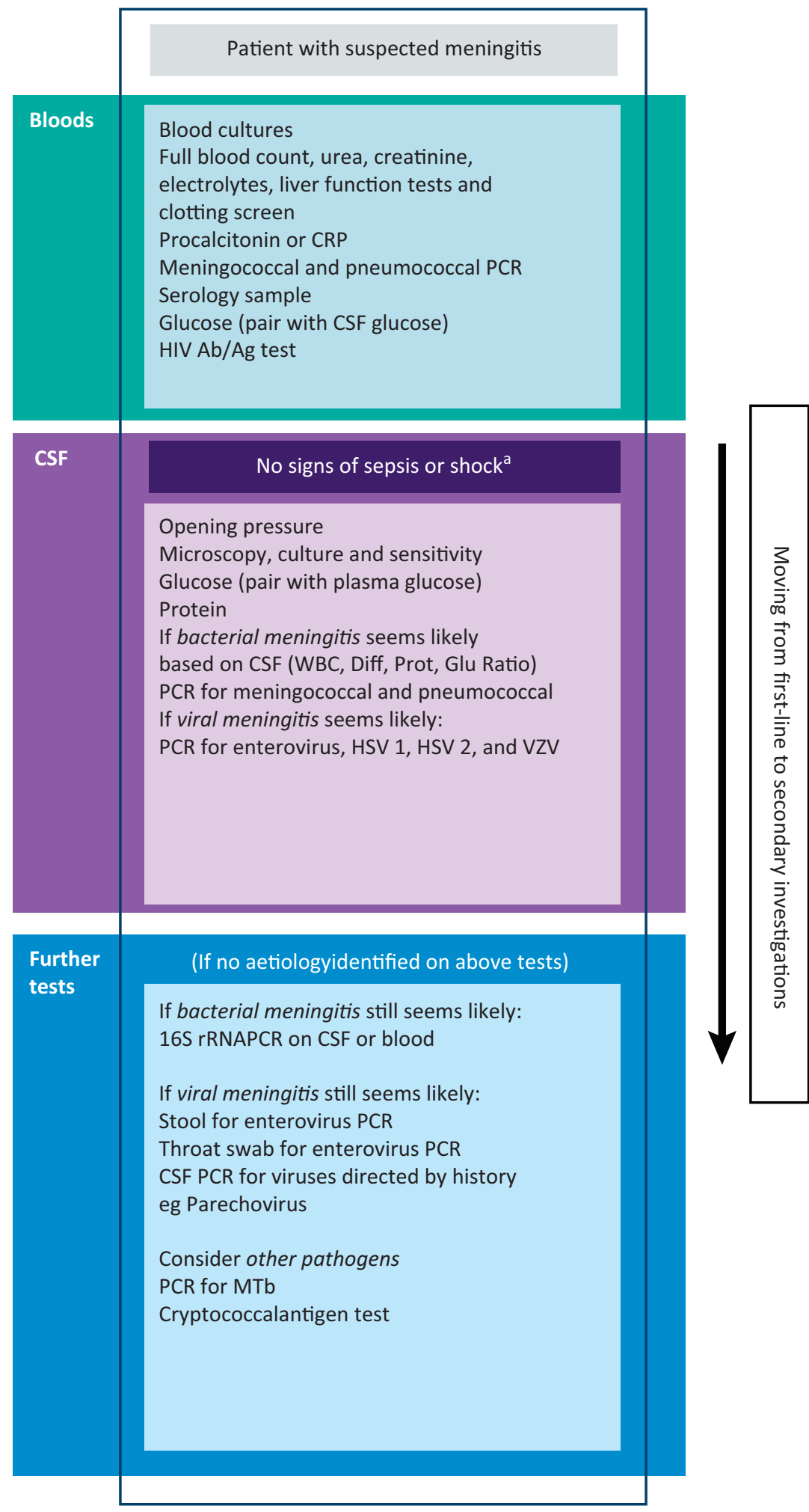

aIf any of the following features are present LP should be delayed:

> Signs of severe sepsis or rapidly evolving rash

$>$ Respiratory or cardiac compromise

$>$ Anticoagulant therapy / known thrombocytopenia

$>$ Infection at the site of LP

$>$ Focal neurological signs ${ }^{b}$

$>$ Presence of papilloedema ${ }^{b, c}$

$>$ Continuous or uncontrolled seizurest

$>\mathrm{GCS} \leq 12^{\mathrm{b}, \mathrm{d}}$

${ }^{c}$ inability to see the fundus is not a contraindication to LP

${ }^{d} L P$ may be safe at lower levels of consciousness

${ }^{\mathrm{b}}$ Neuroimaging should be performed before LP for these indications

Once the patient is stable and if meningitis is likely (with or without sepsis) an LP may still be diagnostically useful, even after several days.

Fig 1. Investigations algorithm. CSF = cerebrospinal fluid; $C R P=C$-reactive protein; Diff = white cell differential; GCS = Glasgow Coma Scale; Glu = glucose (CSF:blood ratio); $\mathrm{HSV}=$ herpes simplex virus; $\mathrm{LP}=$ lumbar puncture; $\mathrm{Mtb}=$ Mycobacterium tuberculosis; $\mathrm{PCR}=$ polymerase chain reaction; Prot $=\mathrm{CSF}$ Protein; VZV = varicella zoster virus; rRNA = ribosomal ribonucleic acid 
Step 1. Suspect pyogenic bacterial meningitis

(assuming Streptococcus pneumonia or Neisseria meningitides)

a. Start ceftriaxone 2 g IV 12-hourlya (or cefotaxime 2 g IV 6-hourly)

b. In patients with probable meningococcal sepsis not treated with ceftriaxone, give a single dose of ciprofloxacin $500 \mathrm{mg}$ orally (to eliminate carriage)

c. In patients at risk for Listeria monocytogenes, such as patients with relative immunocompromise or old age ${ }^{\mathrm{b}}$ ), consider adding in amplicillin/amoxycillin (step 2iiib).

\section{Step 2. Review after Gram stain}

i. Gram-positive diplococci - likely Streptococcus pneumoniae

a. Continue as step 1a (empiric treatment)

b. If the patient visited a country with suspected penicillin resistance ${ }^{a}$

Add vancomycin $15-20 \mathrm{mg} / \mathrm{kg}$ IV 12 -hourly until resistance data available

Alternatively, add rifampicin $600 \mathrm{mg}$ IV (or orally) 12-hourly

Rifampicin should be used instead of vancomycin in renal failure

ii. Gram-negative diplococci-likely Neisseria meningitidis

a. Continue as step $1 \mathrm{a}$

iii. Gram-positive bacilli - suggestive of Listeria monocytogenes

a. Continue as step 1 a

b. Add ampicillin/amoxicillin $2 \mathrm{~g}$ IV 4-hourly

iv. Gram-negative rods - suggestive of Enterobacteriaceae

a. Continue as step $1 \mathrm{a}$

b. Seek specialist advice on local antimicrobial resistance

c. If high suspicion of extended spectrum beta lactamase (ESBL) resistance switch to meropenem 2 g IV 8-hourly

\section{Step 3. Review when culture/PCR results available}

i. Streptococcus pneumoniae confirmed

a. If penicillin sensitive (MIC $\leq 0.06 \mathrm{mg} / \mathrm{L}$ )

Continue as step 1a (or switch to benzylpenicillin 2.4 g IV 4-hourly )

b. If penicillin resistant (MIC >0.06) but cephalosporin sensitive

Continue as step $1 a$

c. If penicillin and cephalosporin resistant

Add vancomycin 15-20 mg/kg IV 12-hourly and rifampicin $600 \mathrm{mg}$ IV/orally 12-hourly

d. If antibiotic sensitivity for organism not known (eg. pathogen PCR data only)

Continue as step 2i (as for Gram-positive diplococci)

e. In patients recovered by day 10 , treatment should stop. Otherwise treat for 14 days

$\mathrm{f}$. In all cases of penicillin or cephalosporin resistance, treat for 14 days

ii. Neisseria meningitidis

a. Continue as step 1a (or switch to benzylpenicillin 2.4 g IV 4-hourly)

b. For patients recovered by day 5 , treatment can stop

iii. Listeria monocytogenes

a. Switch to ampicillin/amoxicillin 2 g IV 4-hourly

b. Alternatively, give co-trimoxazole $10-20 \mathrm{mg} / \mathrm{kg}$ in four divided doses

(of trimethoprim component) in patients with a history of anaphylaxis to beta lactams

c. Treat for at least 21 days

iv. Haemophilus influenzae

a. Continue step $1 \mathrm{a}$

b. Treat for 10 days

v. Enterobacteriaceae

a. Continue as step 2 iv (for Gram-negative rods )

b. Treat for 21 days

Fig 2. Three steps for antibiotic treatment in suspected pyogenic bacterial meningitis. IV = intravenous; MIC = minimal inhibitory concentration 
Few studies have examined outcome in viral meningitis. One recent study, reported one-third of adult varicella meningitis patients (3/9) suffered sequelae. ${ }^{24}$ In our experience, viral meningitis patients can suffer cognitive and psychological sequelae. Headaches occur in one-third of patients. Where there is concern, patients should access neuropsychological services, which can help detect subtle impairments and may facilitate functional recovery. Organisations such as the Meningitis Research Foundation (www.meningitis.org) or Meningitis Now (www. meningitisnow.org), can also provide helpful patient information and advocacy.

\section{Conclusion}

Many meningitis patients in the UK who have a CSF pleocytosis never have a pathogen identified. Clinicians need to remain vigilant and treat suspected bacterial meningitis promptly. However, with viruses being the most common cause of meningitis, rapid diagnosis via PCR can limit unnecessary antibiotic treatment and expedite hospital discharge.

\section{References}

1 McGill F, Heyderman RS, Michael BD et al. The UK joint specialist societies guideline on the diagnosis and management of acute meningitis and meningococcal sepsis in immunocompetent adults. J Infect 2016;72:405-38.

2 Khetsuriani N, Quiroz ES, Holman RC, Anderson LJ. Viral meningitisassociated hospitalizations in the United States, 1988-1999. Neuroepidemiology 2003;22:345-52.

3 Kupila L, Vuorinen T, Vainionpaa R et al. Etiology of aseptic meningitis and encephalitis in an adult population. Neurology 2006;66:75-80.

4 Floren-Zabala L, Chamizo-Lopez F], Eisman-Maraver A et al. [Aseptic meningitis in an adult population. Etiology and utility of molecular techniques in the clinical management of patients]. Enferm Infecc Microbiol Clin 2012;30:361-6.

5 Turtle L, Jung A, Beeching NJ et al. An integrated model of care for neurological infections: the first six years of referrals to a specialist service at a university teaching hospital in Northwest England. BMC Infect Dis 2015;15:387.

6 van de Beek D, de Gans J, Spanjaard L et al. Clinical Features and Prognostic Factors in Adults with Bacterial Meningitis. N Engl J Med 2004:351:1849-59.

7 Stockdale AJ, Weekes MP, Aliyu SH. An audit of acute bacterial meningitis in a large teaching hospital 2005-10. QJM 2011;104:1055-63.

8 Attia J, Hatala R, Cook DJ, Wong JG. The rational clinical examination. Does this adult patient have acute meningitis? JAMA 1999;281:175-81.

9 Durand ML, Calderwood SB, Weber D] et al. Acute bacterial meningitis in adults: a review of 493 episodes. N Engl J Med 1993;328:21-8.

10 Gillespie IA, McLauchlin J, Little CL et al. Disease presentation in relation to infection foci for non-pregnancy-associated human listeriosis in England and Wales, 2001 to 2007. J Clin Microbiol 2009:47:3301-7.
11 Thwaites G, Fisher M, Hemingway C et al. British Infection Society guidelines for the diagnosis and treatment of tuberculosis of the central nervous system in adults and children. $J$ Infect 2009;59:167-87.

12 Moxon CA, Zhao L, Li C et al. Safety of lumbar puncture in comatose children with clinical features of cerebral malaria. Neurology 2016;87:2355-62.

13 Hase R, Hosokawa N, Yaegashi M, Muranaka K. Bacterial meningitis in the absence of cerebrospinal fluid pleocytosis: A case report and review of the literature. Can J Infect Dis Med Microbiol 2014;25:249-51.

14 Spanos A, Jr Harrell FE, Durack DT. Differential diagnosis of acute meningitis. An analysis of the predictive value of initial observations. JAMA 1989;262:2700-7.

15 Brouwer MC, Thwaites GE, Tunkel AR, van de Beek D. Dilemmas in the diagnosis of acute community-acquired bacterial meningitis. Lancet 2012;380:1684-92.

16 Nigrovic LE, Kuppermann N, Macias CG et al. Clinical prediction rule for identifying children with cerebrospinal fluid pleocytosis at very low risk of bacterial meningitis. JAMA 2007;297:52-60.

17 Heinsbroek E, Ladhani S, Gray S et al. Added value of PCR-testing for confirmation of invasive meningococcal disease in England. J Infect 2013;67:385-90.

18 Newcombe J, Cartwright K, Palmer WH, McFadden J. PCR of peripheral blood for diagnosis of meningococcal disease. J Clin Microbiol 1996;34:1637-40.

19 Morales Casado MI, Moreno Alonso F, Juarez Belaunde AL et al. Ability of procalcitonin to predict bacterial meningitis in the emergency department. Neurologia 2016;31:9-17.

20 Vikse J, Henry BM, Roy J et al. The role of serum procalcitonin in the diagnosis of bacterial meningitis in adults: a systematic review and meta-analysis. Int J Infect Dis 2015;38:68-76.

21 Proulx N, Frechette D, Toye B, Chan J, Kravcik S. Delays in the administration of antibiotics are associated with mortality from acute bacterial meningitis. QJM 2005;98:291-8.

22 Dellinger RP, Levy MM, Rhodes A et al. Surviving sepsis campaign: international guidelines for management of severe sepsis and septic shock: 2012. Crit Care Med 2013;41:580-637.

23 Alam N, Oskam E, Stassen PM et al. Prehospital antibiotics in the ambulance for sepsis: a multicentre, open label, randomised trial. Lancet Respir Med 2018;6:40-50.

24 Kaewpoowat Q, Salazar L, Aguilera E, Wootton SH, Hasbun R. Herpes simplex and varicella zoster CNS infections: clinical presentations, treatments and outcomes. Infection 2016;44:337-45

25 Tunkel AR, Hartman BJ, Kaplan SL et al. Practice guidelines for the management of bacterial meningitis. Clin Infect Dis 2004:39:1267-84.

Address for correspondence: Dr Michael J Griffiths, Department of Clinical Infection, Microbiology and Immunology, Institute of Infection and Global Health, University of Liverpool, 8 West Derby Street, Liverpool L69 3BE, UK.

Email: griffmj@liv.ac.uk 